\title{
Recognition and Positioning Method of Rice Seedlings Based on Machine Vision
}

\author{
Jiang Yu ${ }^{1,2}$, Cui Hongwei ${ }^{1}$, Qi Long ${ }^{1,3 *}$, Zheng Wenhan ${ }^{1}$ and Zhao Liulin ${ }^{1}$ \\ ${ }^{1}$ College of Engineering, South China Agricultural University, \\ Guangzhou 510462, China \\ ${ }^{2}$ Modern Educational Technology Center, South China Agricultural University, \\ Guangzhou 510462, China \\ ${ }^{3}$ Southern Regional Collaborative Innovation Center for Grain and Oil Crops in \\ China, Changsha 510462, China \\ *qilong@scau.edu.cn
}

\begin{abstract}
Recognition and positioning of rice seedlings is a precondition of mechanical intra-row weeding. An innovative method to recognize and position rice seedlings based on machine vision was proposed in this paper. First, RGB images of rice were captured with a system that analyzes the related factors affecting image quality under natural light condition, where the related factors mainly included whether there was water and whether there was shading in the paddy fields. It indicated that the image quality could be improved by shading the captured RGB images. The original RGB images were processed by image processing methods including transferring color image to gray image by identifying the intensity of green components, filtering noises using the PointFilter toolbox and segmentation by automatic threshold. Through measuring the number of connected regions with ExtractBlobs toolbox, the whole rice seedling was extracted in the detection circular domain. The maximum number of connected regions was 6 in the detection domain. The centers of a fixed number connected regions were used to position the center of rice seedlings and 6 center coordinates were obtained for each image, which was named as fixed number of connected regions method. A new position method that compares different points and dynamic positioning, was proposed based on the method of fixed number of connected regions. Using this method, the center with minimum average was set as the positioning center by comparing the average offset of six centers. The method of comparison of different points and dynamic positioning was more accurate. The experiments were carried out both in soil bin and field conditions. Results in soil bin showed that the positioning accuracy was $8 \mathrm{~mm}$ for the 50 samples captured with water layer thickness of $1.5 \mathrm{~cm}$ and $9.1 \mathrm{~mm}$ for the 50 samples with anhydrous condition. In the field tests, the protection domain was determined considering both the diameter of stem base of rice seedlings and agronomic mechanized transplanting requirements. Field test results showed that the positioning accuracy was $5.4 \mathrm{~mm}$ and correctness was $90 \%$ for the 50 samples with water layer thickness of $1.5 \mathrm{~cm}$, and the processing time for one image was $8.5 \pm 1.5 \mathrm{~ms}$. The experimental results showed that the proposed location method of rice seedling center meets the requirements of intelligent mechanical intra-row weeding both in accuracy and processing speed.
\end{abstract}

Keywords: rice seedlings, machine vision, mechanical weeding, intra-row weeding, recognition and position

${ }^{*}$ Corresponding Author 


\section{Introduction}

How to avoid damaging rice seedlings and conduct selective weeding poses the greatest difficulty to the mechanical weeding. Mechanical rice-transplanting, theoretically, could make spacing in the rows uniform, yet it's another story due to different amounts of seedlings taken per unit area, leaking or floating seedlings. Thus, the accurate recognition and positioning of rice seedlings shall come before the mechanical weeding in the paddy fields.

Generally, crop automatic recognition and positioning often requires the machine vision technology. However, it makes harder to conduct researches on and apply the field intelligent mechanical-weeding equipment due to the complexity of the image background, similarity in the plants architecture and weeds, and many environmental factors. Among all efforts, Tillett [2] et. al., employed two-dimensional wavelet technology in combination with Kalman's tracking algorithm to recognize the targeted lettuce and obtain the positioning information. With all these decision-making data available, the weed control component completed the intral row weeding. Beyond that, Astrand et. al., [3], in laboratory conditions, selected three characteristic values of color images of the crop, and then set up $\mathrm{K}$ nearest neighbor classification model to identify the targeted beet and to obtain its positioning information. In addition, Chun-long zhang et. al., [4], with the goals of the minimum time consumed and the maximum inclusive accuracy, took advantage of parameters combination of image row pixel histogram to determine the one-dimension coordinate that the robot moved forward between seedlings with positioning errors of $\pm 5 \mathrm{~mm}$. Besides, Lian $\mathrm{Hu}$ et. al., [5] used Ostu image segmentation and morphological operation method to recognize cotton seedlings and lettuce seedlings in the super green space. Then, the crops' positioning information was obtained in accordance with the pixel ranks' accumulation combined with the center of mass connecting the green seedlings' domain. Against the backdrop of complex environment, there were still errors in positioning crop using canopy image through the machine vision technology.

The above-mentioned researches adopted the machine vision technology in combination with image-processing algorithms to recognize and locate crops [6-7]. However, crops identified and located researches focused on vegetables in dry fields and its canopy morphology is relatively simple. Nevertheless, as for rice seedlings, it's not easy, technically, to identify and locate them with the technology of machine vision due to complex background environment, reflection of water layer and complicated morphological features of rice seedlings.

This study aimed to build an image acquisition system featuring higher different gray value between rice seedlings and the background in natural light through exploration on the related factors affecting the quality of paddy field imaging. Beyond that, images were also processed through the integrated application of color image transferred to gray image, noise filter and automatic threshold. Then, a seedling area was marked, and the number of connected areas used to extract the complete rice seedlings was gathered; afterwards, different points of comparison and the method of dynamic positioning were proposed; at the end, in light of the growth characteristics of pointed planting, stem base beam and loose canopy, protected domain of rice seedling were judged according to stem-base diameter.

\section{Materials and Methods}

\subsection{Rice Seedlings Cultivation}

Rice variety tested as samples was Hua Hang Si Miao. Rice seedlings were cultivated in the greenhouse $\left(113.35^{\circ} \mathrm{E}, 23.16^{\circ} \mathrm{N}\right)$, with $60 \sim 70 \%$ relative humidity inside, and with average temperature $32{ }^{\circ} \mathrm{C}$ and $24{ }^{\circ} \mathrm{C}$ day and night respectively. Rice seeds were 
soaked 24 hours for disinfection, and then were rinsed and sprouted. Afterwards, those seeds were potted as experiments, totaling 100 pots with 4 6 grains in each pot. Meanwhile, fertilization and watering still followed the conventional managerment. When the average height of rice seedlings was up to about $260 \mathrm{~mm}$ with canopy average diameter about $13 \mathrm{~cm}$, those potted rice seedlings were transplanted to soil bins. Intrarow spacing is $120 \sim 150 \mathrm{~mm}$ and inter-row spacing is $300 \mathrm{~mm}$. Relative humidity where the soil bin was placed was $65 \sim 75 \%$ with average temperatures of $30{ }^{\circ} \mathrm{C}$ and $23{ }^{\circ} \mathrm{C}$, day and night respectively. The soil in each bin simulating paddy field was from the paddy soil with weeds scattered.

\subsection{Image Acquisition}

2.2.1. Image Acquisition System: Image acquisition system was mainly composed of an intelligent color camera (ISM1400C, COGNEX, US), lens (M0814-MP, COMPUTAR, Japan), software (Insight, explore, COGNEX, US), a computer (B470, lenovo, China), the soil bin (length $\times$ width $\times$ height is $200 \times 60 \times 20 \mathrm{~cm}$ ) shading devices and so on, as shown in Figure 1. To be more specific,the camera resolution was $640 \times 480$ pixels with focal length of $8 \mathrm{~mm}$ and an aperture of F1.4 - F16C. Beyond that, the aperture and focal length need to be manually adjusted and then fixed. Besides, the frame of shading devices were made of aluminum alloy, and the shading cloth were made of the polyester fiber.

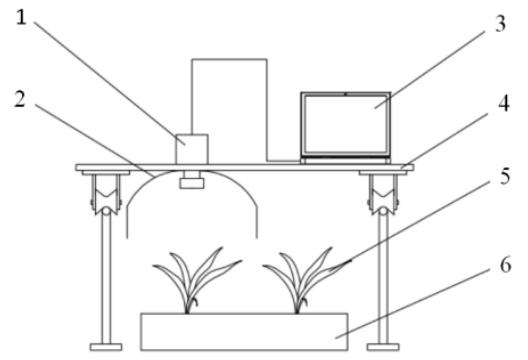

1. Intelligent camera 2. Shading devices 3. Computer 4. Mobile platform 5. Rice seedling 6. Soil bin

Figure 1. Image Acquisition System

2.2.2. The Environmental Conditions of Image Acquisition: In a bid to make obvious the difference between the rice seedlings and graying background and to make the image less noisy, relevant factors impacting the quality of images needed to be adjusted [8]. Among these factors, it should be taken into consideration that whether the imaging area was watery, and whether the imaging area was shading. In this study, the shading of imaging area meant that shading devices put rice-seedlings imaging area in lightscattering area without water reflection and bright spots. Besides, the shading device layout was shown in Figure 1. Thus, in order to figure out how the above-mentioned two factors influenced the quality of images, the image acquisition was conducted respectively under four types of condition, namely, anhydrous and no shading, water and no shading, anhydrous and shading, water and shading. If there was water, the thickness of water was around $15 \mathrm{~mm}$, and the image acquisition results were shown in Figure 2.

Comparing Figure 2a, and $2 \mathrm{c}$, it was clear that against the backdrop of sunshine and soil reflection in paddy fields, the difference between paddies and soil gray level was not obvious; on the other hand, under the shading condition, the difference between paddies and soil gray level was obvious, which made it easy to segment the images. When there was no shading in paddy field, the water would reflect the mobile platform, the sky, clouds and other objects. That explained why there were more background noises in Figure 2, and thus the following images were processed in a more difficult way. In 
contrast, the images quality would be much better under shading conditions. Therefore, this study conducted the image acquisition under shading conditions.

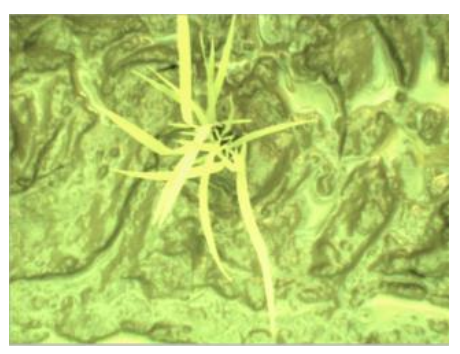

(a) The Condition of Anhydrous and No Shading

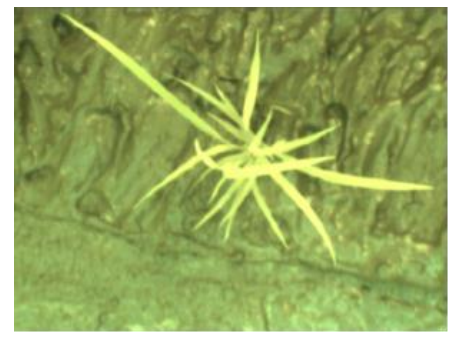

(c) The Condition of Anhydrous and Shading

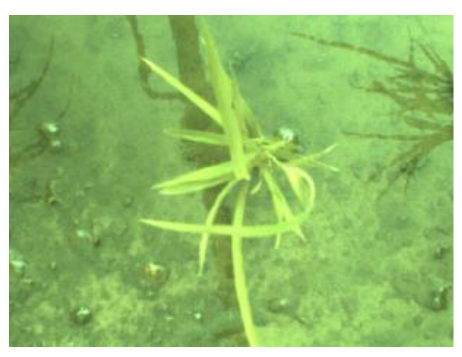

(b) The Condition of Including Water and No Shading

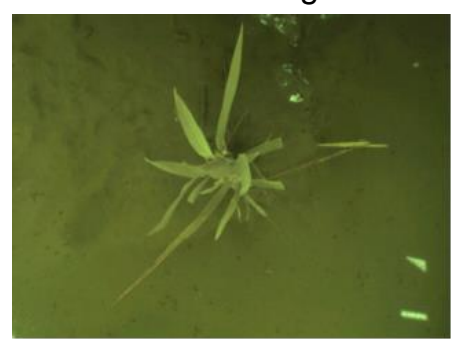

(d) The Condition of Including Water and Shading

\section{Figure 2. Images Captured under Different Conditions}

2.2.3. Image Acquisition Steps: Image acquisition steps were as follows: 1) to adjust the camera position to make rice seedlings in the central image; 2 ) to adjust the focal length and aperture of the camera to make the image clear; 3) to adjust water-layer thickness in the soil bin to about $15 \mathrm{~mm}$; 4) to capture and save the image; 5) to drain out the water in the soil bin and repeat the fourth step ; 6) to transplant the next batch of paddies ready to be collected into the soil bins after completion of collection of image samples. Afterwards, soil bin stand still for 15 minutes, then following third, fourth and fifth steps again.

200 image samples with and without water shading were chosen, with 100 images in each condition. Among them, 50 images for each condition were used for method-based analysis while the rest of samples were used for the test of positioning accuracy.

\subsection{Image Segmentation}

Due to the targeted rice seedlings in green, the color images were transformed into the green component of gray image, thus making it easier to separate the rice seedlings apart from the background in the image. Therefore, the processed images could highlight the gray value of rice seedlings, with grayed result shown in Figure 3.

To reduce the noise in the background, it was necessary to filter interference pixels within the scope of low pixel values. By setting pixel value range necessary to be filtered, clamp function filtered the pixel within the scope of the fixed pixel values. And its basic principle was: the clamp function processed image gray value according to the following formula:

$$
F(x, y)=\max \{f(x, y), K\}
$$

In this formula, $f(x, y)$ was the gray levels of pixels, and $K$ was the set gray value of the boundaries, and $F(x, y)$ was the gray value after those images were processed. Under the auspices of PointFilter tools in the clamp function, $K$ value could be adjusted to filter the noise of the image with the results shown in Figure 3. 


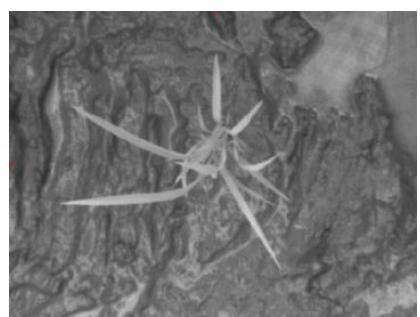

Figure 3. Gray Image

In the image acquisition, the changes in such factors as light intensity, height of rice seedlings, and the object distance would affect gray value distribution in images. Therefore, it was impossible to make the image segmentation through the fixed threshold method. This study adopted Ostu method, an image thresholding algorithm, to automatically specify the threshold to have the binarization processing for images. In detail [9-10], Ostu method automatically performed clustering-based image thresholding, $T_{h}$. Therefore, the rice seedlings and background in the image could be set apart in accordance with the following formula.

$G(x, y)=\left\{\begin{array}{cc}255, & g(x, y) \geq T_{h} \\ 0, & g(x, y)<T_{h}\end{array}\right.$

In the formula, $g(x, y)$ was the gray value of pixels, and $g(x, y)=0$ was the background (in black), and $g(x, y)=255$ meant rice seedlings, weeds or noise (in white).

Based on the PointFilter tool to filter the noise of the image, different $K$ values would result in difference in binary images of rice seedlings [11], as shown in Figure 4. In detail, when taking smaller $K$ values, there were more noises in the images. On the other hand, when taking larger $K$ values, there was impressive lack of effective pixels in images. After comparing many images, it was found that when $K$ value equaled 130, the rice seedlings in binary images were complete with less noises. That explained why this study set $K$ value at 130 .

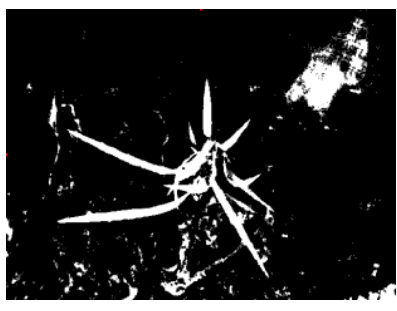

(a) $K=120$

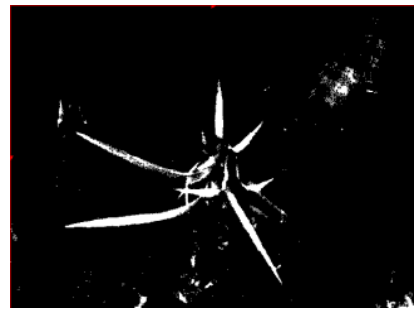

(c) $K=140$

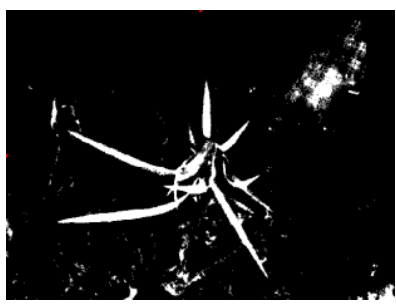

(b) $K=130$

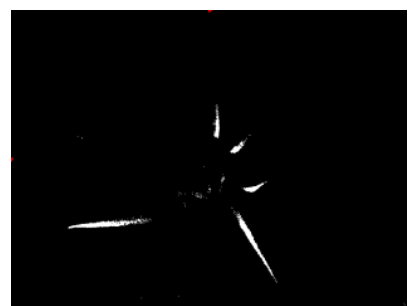

(d) $K=150$

Figure 4. Binary Image of Rice Seedlings with Different $K$

Figure 5a, showed the processing results of the automatic threshold after graying RGB images while Figure 5b, showed the processing results of the automatic threshold after filtering noise through PointFilter tools with $\mathrm{K}$ value at 130 after graying RGB. Thus, it could be detected that, through contrasting these two figures, noise could effectively 
become less due to the PointFilter tools, as shwon in Figure 5b.

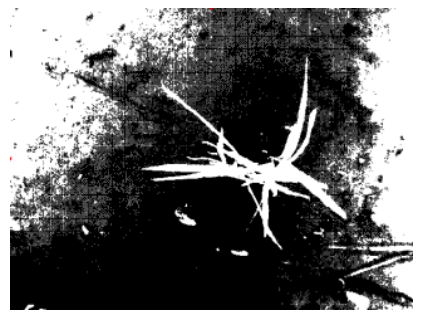

(a) Binary Image without PointFilter

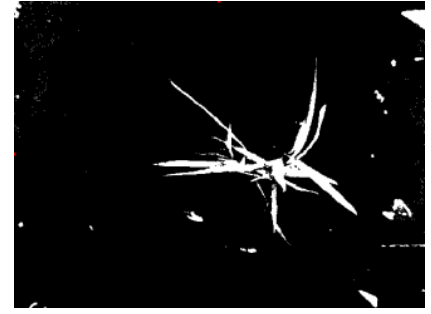

(b) Binary Image with PointFilter

Figure 5. Comparison of Processing Results with PointFilter or Not

\subsection{The Positioning Method for Rice Seedlings}

2.4.1. Blob Algorithm: In the binary images, the rice seedlings and part of the noise were composed of white-pixels connected area. Through calculating the center coordinate of the connected area belonging to rice seedlings, then the center coordinate for positioned rice seedlings was obtained. Based on Blob algorithm to calculate center coordinates $\left(x_{0}, y_{0}\right)$ of the connected area, the principle was that $R(p, q)$ step interval of connected regions was [12-13]:

$$
M_{p q}=\sum_{x \in R} \sum_{y \in R} x^{p} y^{q} f(x, y)
$$

In the formula, point $(x, y)$ was located in the connected area or on the border. Therefore, $M_{00}$ in the connected area represented pixel points for the connected area. The step intervals for $\mathrm{X}$ direction and $\mathrm{Y}$ direction respectively were:

$M_{10}=\sum_{x} \sum_{y} x f(x, y)$

$M_{01}=\sum_{x} \sum_{y} y f(x, y)$

The center coordinates was as follows:

$$
\left\{\begin{array}{l}
x_{0}=M_{10}(R(x, y)) / M_{00}(R(x, y)) \\
y_{0}=M_{01}(R(x, y)) / M_{00}(R(x, y))
\end{array}\right.
$$

In the formula, $R(x, y)$ was the connected area, and $f(x, y)$ represented pixel values of the coordinates. Based on ExtractBlobs tools in Blob algorithm, it was easier to obtain the center coordinate of the connected area in the binary images. That explained why the extraction of the connected area in the images were all completed by ExtractBlobs tool.

2.4.2. Calibration of the Center of Rice Seedlings Against the Pixel Coordinates: The calibration of the center of rice seedlings was a key to measure whether the positioning was accurate. In this study, the exact coordinate of the center of rice seedlings against the pixel coordinates was gained by manual calibration in the following way. Prior to image acquisition, a white bar was inserted in the center of the rice seedlingss, as shown in Figure 6a. Then, after image binarization, the center coordinate of the white-bar connected area was selected as the center ones of pixel coordinates of rice seedlings, as shown in Figure 6b. The calibrated center was shown on the top left corner in Figure 6b. 


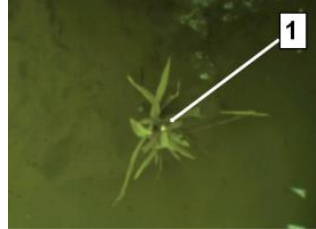

1. Benchmarking

(a) RGB Image

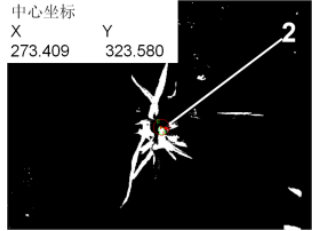

2. Center of calibration

(b) Image of Central Calibration

Figure 6. Center Calibration

2.4.3. Positioning-Method Analysis: 1) To mark the seedling area. In binary images, the pixel values for some weeds, bright spots and rice seedlings were 1, which resulted in more noise in the images. When extracting rice seedlings within the whole image, the positioning accuracy of rice seedlings was impacted. To ensure less noisy interference with the rice seedlings, the domain of detection was marked in the center of the image. That meant that visual identification system was used to extract the area of rice seedlings in the course of machinery weeding in paddy fields.

The width of rice seedlings crown covered about $3 / 4$ width of the image, with radius of about 180 pixels. Accordingly, the radius of the domain of detection was set at 210 pixels (when rice seedlings were fully extracted, the radius value was set greater than 180 pixels). As shown in Figure 7, the circle in the figure meant the domain of detection.

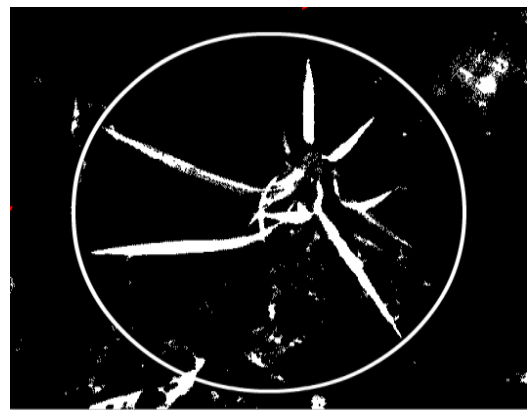

Figure 7. Domain of Detection

2) To calculate the connected area to extract the whole-cave rice seedlings. The fold leave of rice seedlings, weeds and bright spots would impact the process of calculating the connected area to extract the whole-cave rice seedlings in domain of detection [14-15]. Thus, when extracting the connected area of rice seedlings in the domain of detection, it was assumed that $\mathrm{n}$ meant the number of connected areas extracting rice seedlings. When $\mathrm{n}$ value was too small, the rice seedlings extraction was incomplete; When the $\mathrm{n}$ value was too large, the extraction results included more noises, as shown in Figure 8. But these two scenarios were not conducive to the center positioning. Therefore, the rice-seedlings-extraction experiment was needed to measure $\mathrm{n}$ value. Based on ExtractBlobs tool, the rice-seedlings connected areas with less than 100 filtered pixel were extracted among those 100 images using for method analysis. The extraction results were shown in Table 1. 


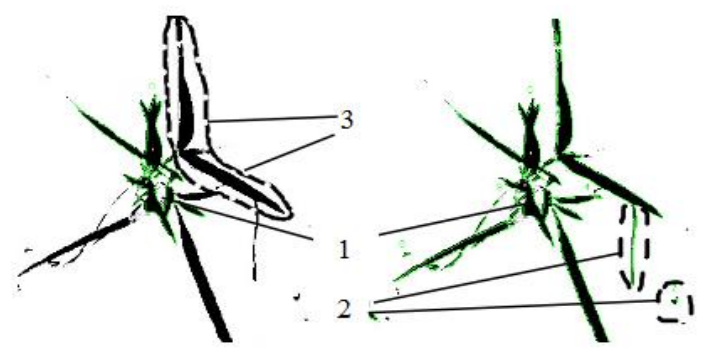

1. Rice Seedlings 2. Noises 3. Rice Seedlings not being Extracted

(a) Image of Uncompleted Rice Seedlings (b) Image of Rice Seedlings and Noises

Figure 8. Extracting Results of Rice Seedlings

Table 1. Number of Connected Region of Extracting Completed Rice Seedlings

\begin{tabular}{cc}
\hline Number of connected region & Number of sample \\
\hline 1 & 62 \\
2 & 86 \\
3 & 94 \\
4 & 97 \\
5 & 98 \\
6 & 100 \\
\hline
\end{tabular}

The Table 1, showed that when the number of connected area was 6 , the rice seedlings in 100 image samples were extracted. On the other hand, when the number of connected areas was more than 6 , extraction results contained more noises. Consequentially, this study set the maximum value of $n$, the number of connected areas for the extraction of whole-cave rice seedlings, at 6 .

3) To position the fixed connected-area number. To extract the connected areas of the domain of detection from 100 image samples, those samples were arranged according to the pixel size in the descending way. Meanwhile, the geometric center coordinates including the connected areas were extracted, namely the center coordinates of the connected areas. Based on the order of the connected areas, $\mathrm{j}$ connected areas were selected to calculate the center of rice seedlings. When the number of connected areas used for the calculation of the center of rice seedlings was less than or equal to the number of connected areas used to extract the whole-cave rice seedlings, namely $0<j \leq n \leq 6$, the calculation formula was defined as Formula 7.

$\begin{cases}X_{i j}=\frac{x_{1}+x_{2}+\ldots+x_{j}}{j} & j=1 \ldots n \\ Y_{i j}=\frac{y_{1}+y_{2}+\ldots+y_{j}}{j} & j=1 \ldots n\end{cases}$

In this formula, $\left(\mathrm{X}_{\mathrm{ij}}, \mathrm{Y}_{\mathrm{ij}}\right)$ represented the positioning center of center coordinates of $\mathrm{j}$ connected areas in the first image, with $\left(\mathrm{x}_{\mathrm{j}}, \mathrm{y}_{\mathrm{j}}\right)$ as the center coordinates of the connected areas. When the number of connected areas used for the calculation of the center of rice seedlings was more than the number of connected areas used to extract the whole-cave rice seedlings, namely $n<j \leq 6, X_{i j}$ equaled $X_{i n}$, and $Y_{i j}$ equaled $Y_{i n}$. Thus, when $j$ value was set between 1 6, each image could obtain six center coordinates of rice seedlings.

4) To compare between different points and position dynamically. As there was deviation between the positioning center for the fixed number of connected areas and the calibration center, this deviation was defined as the coordinate offset value with the following calculation formula: 
$L_{i j}=\sqrt{\left(X_{i j}-X_{i 0}\right)^{2}+\left(Y_{i j}-Y_{i 0}\right)^{2}} \quad i=1,2 \ldots 50$

In the formula, $\left(\mathrm{X}_{\mathrm{ij}} \mathrm{Y}_{\mathrm{ij}}\right)$ represented the positioning coordinates of rice seedlings in the first image, and $\left(\mathrm{X}_{\mathrm{i} 0}, \mathrm{Y}_{\mathrm{i} 0}\right)$ represented the calibration coordinates of rice seedlings in the first image, and $\mathrm{L}_{\mathrm{ij}}$ represented the offset value of coordinates in the first image. Each positioning center corresponded to a coordinate offset value, thus with six coordinate offset values in each image.

As the analysis was made on the number of the connected areas used to extract the complete rice seedlings and on the positioning results for the number of fixed connected areas, it was concluded that, in different images, different numbers of connected areas were used at the minimum center of coordinate offset values. In order to pick up the positioning center of the coordinate offset values, following steps were needed to have the positioning: six centers from positioning results of fixed connected areas served as the benchmark centers in order to calculate the average offset values between the benchmark center and the remaining five centers. Then, each image obtained six average values, among which the center coordinate matching the minimum value represented the center of rice seedlings positioned according to this method. For instance, the six average values from one image were $\overline{\mathrm{L}_{11}} 、 \overline{\mathrm{L}_{12}} 、 \overline{\mathrm{L}_{13}} 、 \overline{\mathrm{L}_{14}} 、 \overline{\mathrm{L}_{15}}, \overline{\mathrm{L}_{16}}$. If $\overline{\mathrm{L}_{11}}$ was less than min $\left(\overline{L_{12}}, \overline{\mathrm{L}_{13}}\right.$, $\left.\overline{\mathrm{L}_{14}}, \overline{\mathrm{L}_{15}}, \overline{\mathrm{L}_{16}}\right)$, the center coordinate of positioned rice seedlings were $\left(\mathrm{X}_{1}, \mathrm{Y}_{1}\right)=\left(\mathrm{X}_{11}, \mathrm{Y}_{11}\right)$. In detail, $\left(\mathrm{X}_{1}, \mathrm{Y}_{1}\right)$ meant the positioning center under this method in the first image. This above-mentioned method was comparison between different points and dynamic position.

5) To compare positioning methods. In order to compare the two positioning methods, the coordinate offset value between positioning centers was calculated under two methods from 100 image samples, among which the maximum value was 48.3 pixels. In a bid to have a better positioning result, the offset value was set within the range of $0 \sim 50$ pixels, and was divided into five equal parts. Then, samples were calculated in each range as shown in Table 2.

\section{Table 2. Sample Number of Two Kind of Positioning Methods in Different} Interval

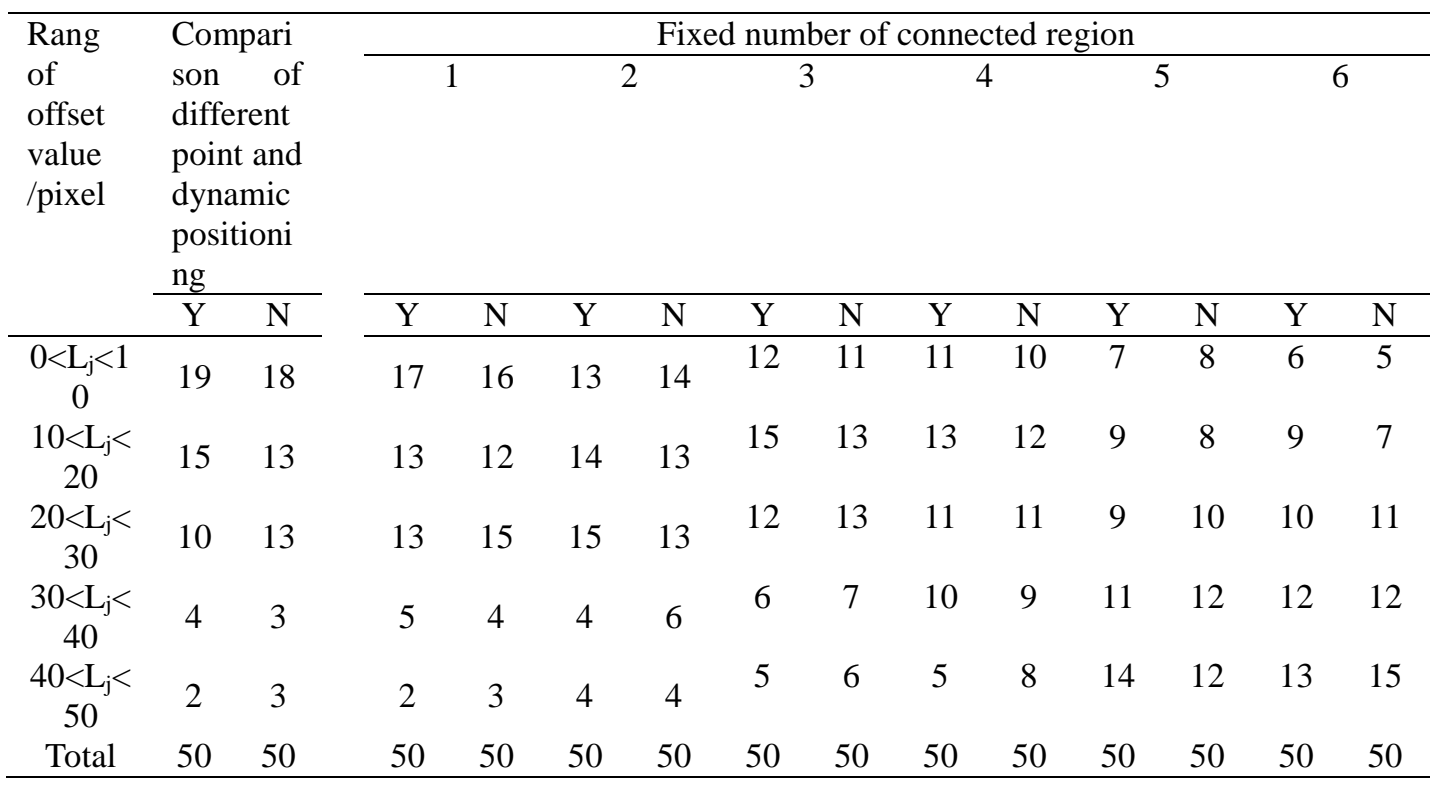

Note: In Table 2, Y represents the condition of including water, $\mathrm{N}$ represents the condition of anhydrous. 
The Table 2, showed that within the range of smaller offset values, samples under the positioning method of comparison between different points and dynamic position were more than the samples under the positioning method of fixed connected areas, and the former's positioning result was more accurate than the latter's.

\section{Experiment and Analysis}

\subsection{Soil Bin Testing and Analysis}

100 images (with or without water, with 50 copies in each condition) were centrally positioned in accordance with the above-mentioned method. Then, the positioning coordinates and the calibration coordinates have transformed from pixel coordinates to actual coordinates and calculated the actual offset value of the positioning center coordinates $\delta$ i. Positioning accuracy was represented by the root-mean-square error (RMSE) with the following formula to calculate RMSE [16]:

$$
\sigma=\sqrt{\frac{\sum_{i=1}^{50} \delta_{i}^{2}}{50}}
$$

In this formula, $\sigma$ represented the root-mean-square error, meaning the positioning accuracy. Besides, $\delta$ i represented the coordinate offset value in the first image. The positioning results of soil bin sampling image were shown in Table 3.

Table 3. Positioning Results of Samples Captured in Soil Bin

\begin{tabular}{cc}
\hline conditions & positioning accuracy $/ \mathrm{mm}$ \\
\hline including water & 8 \\
anhydrous & 9.1 \\
\hline
\end{tabular}

The Table 3, showed that the positioning accuracy was $8 \mathrm{~mm}$ when the images were with water, while the the accuracy was $9.1 \mathrm{~mm}$ when the images were without water. The soil bin testing position error mainly came from the changes in the light intensity around the soil bin which lead to the changes in the amount of light from the camera. As a result, the graying value of the images was impacted, and the gray value of the binary images was represented by 0 and 1 pixels. Consequentially, the changes in the light intensity impacted the distribution of 0 and 1 pixels in the binary images, then standing in the way of the identification and location of rice seedlings.

The contrast in positioning accuracy showed that the positioning could be more accurate if the image was with water. Following factor mainly contribute to this higher accuracy [17-19]: Reflectance water $<$ Reflectance soil $_{\text {r }}<$ Reflectance $_{\text {canopy. That meant that }}$ the difference in graying values of the water and rice canopies would be higher in the image, which would be conducive to the segmentation between the rice seedlings and background image and make the positioning more accurate. As a result, the field image samples were collected in water, shading conditions.

\subsection{The Field Testing and Analysis}

When the intra-row mechanical weeding was conducted, there would be areas that prevent the seedlings from damage. This area served as the protected domain of rice seedlings. The area covered by machines and tools in the process of weeding meant weed coverage area between seedlings. The increase in protected domain could be beneficial to the reduced rates in hurting seedlings whereas it would also decrease the weed coverage area and reduce the weeding rates. Therefore, it needed to set a appropriate range of protected domain in order to reduce the hurting rates and guarantee the weeding rates. 
When the field test was conducted in agricultural science research institute of Zhaoqing city, Guangdong province, the variety of rice seedlings was Yongfeng 9802. 12 days after the mechanical transplanting of rice seedlings, the field test was carried out. At that time, the height of rice seedlings was about $200 \mathrm{~mm}$ with 5 seedlings per cave and with $15 \mathrm{~mm}$-thick water in the paddies. Finally, 50 sampling images were collected with echinochloa crusgalli and rotala indica in the paddies.

The diameters of the canopy and stem-base parts of 50 rice seedlings used for image acquisition were measured with results shown in Figure 9.

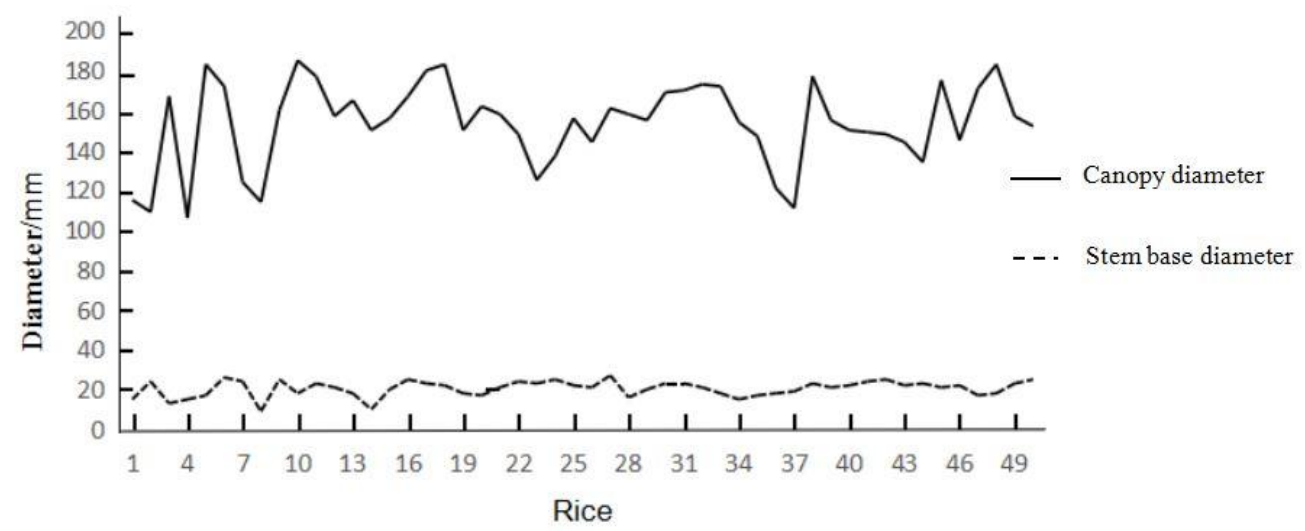

Figure 9. Diameter Distribution of Stem Base and Canopy of Rice Seedlings

Figure 9, showed that the distribution curve of stem-base diameter of rice seedlings were relatively stable while the distribution curve of canopy diameter of rice seedlings were quite volatile. Then, the error in marking the protected domain against the canopy was higher. In weeding between seedlings, weeding components mainly worked for the stem-base parts of seedlings. As a result, protected domains should be marked out according to the stem-base diameter. Besides, practices told us that the planting distance between rice seedlings mechanically transplanted was $120 \sim 170 \mathrm{~mm}$. Taking into consideration the stem-base diameter, planting area, weeding rates, hurting rates, the study set the diameter of the protected domain twice as large as the stem-base diameter of rice seedlings. The area of stem-base parts of rice seedlings and the protected domain were shown in Figure 10.

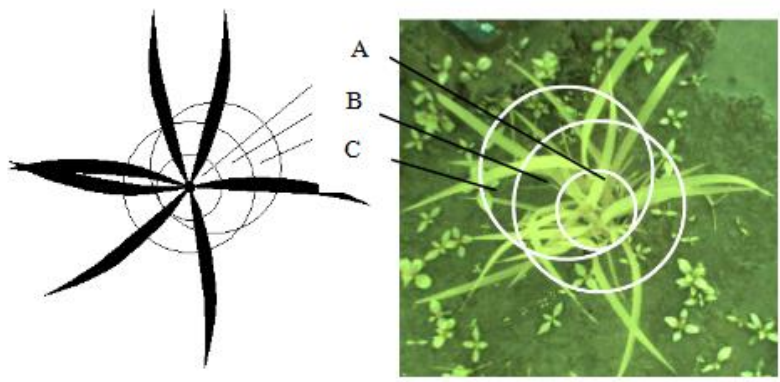

\section{Figure 10. Stem Base and Protected Domain of Rice Seedling}

In Figure 10, Circle A meant the stem-base area of rice seedlings while Circle B meant the protected domain of rice seedlings centering on the calibration which was referred to as calibrated protection area. Besides, Circle $\mathrm{C}$ meant the protected domain of rice seedlings centering on the image positioning which was referred to as positioned protection area. Among them, Circle A and Circle $B$ were concentric, and $2 R_{A}=R_{B}=R_{C}$, and circle $\mathrm{C}$ center was located in the boundary circle $\mathrm{B}$. When the positioning center 
deviated to boundary of the stem-base area of rice seedlings, the Circle $\mathrm{C}$, the positioned protection area, was tangent with Circle A, the stem-base area of rice seedlings which allowed the mechanical weeding not to hurt the seedlings. On the other hand, when the positioning center deviated out of the stem-base area of rice seedlings, the positioned protection area only included some or no seedlings. The plant area contained rice seedlings, and thus weeding coverage between seedlings possibly contained rice seedlings. Thus, to rule out this possibility, weeding coverage area was identified as the rice-plant area by default. To sum up, when the positioning deviated out of stem-base area, the weeding coverage area included rice seedlings, thus hurting seedlings in mechanical weeding.

The diameters of the stem-base area of 50 rice seedlings used for field image acquisition were measured with results shown in Table 4.

Table 4. Distribution of Diameter at Stem Base

\begin{tabular}{cc}
\hline Range of diameter/mm & Number of sample \\
\hline $0-10$ & 14 \\
$10-20$ & 17 \\
$20-30$ & 19 \\
\hline
\end{tabular}

The Table 4, showed in the field the stem-base diameter was less than $30 \mathrm{~mm}$. Therefore, the protected domain of rice seedlings centered on rice seedlings to form a circle with diameter of $60 \mathrm{~mm}$. To sum up, in the field, when the offset value of the positioning center of rice seedlings was less than or equal to $15 \mathrm{~mm}$, the positioning was accurate, and mechanical weeding hurt no seedlings.

In the local diagram of the field image positioning results as shown in Figure 11, the circle meant the protected domain, and X meant the center of the positioning. On the other hand, the field-image positioning results was shown in Table 5. In detail, the positioning accuracy represented the percentage of well-positioned samples in the total samples. The processing period of single image was measured against the software of insight explore.

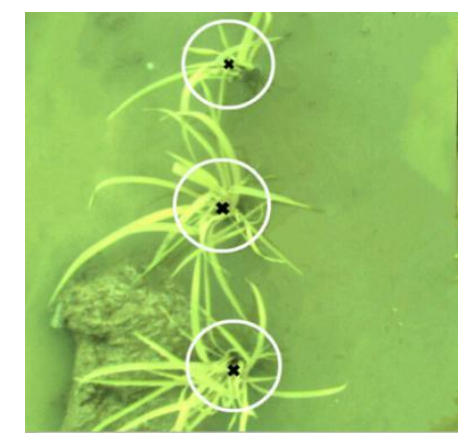

Figure 11. Image of Positioning Results

Table 5. Positioning Results of Samples Captured at Field

\begin{tabular}{ccc}
\hline Positioning accuracy/mm & Accurate rate of & Processing time of one \\
& localization/\% & image/ms \\
8.6 & 90 & $8.5 \pm 1.5$ \\
\hline
\end{tabular}

The Table 5, showed that the positioning accuracy of field images in the filed was 8.6 $\mathrm{mm}$ with the $90 \%$ positioning accuracy. It took $8.5 \pm 1.5 \mathrm{~ms}$ to process a single image. The reason why the positioning accuracy was impacted was that in the process of acquiring field images, the height and shape of weeds and echinochloa crusgalli in the field were quite similar to rice seedlings, and they were easy to be mistakenly identified as rice 
seedlings, which affected the positioning accuracy.

\section{Conclusion}

This article employed machine vision technology to focus on the recognition and position of rice seedlings, and soil bins and field tests were conducted respectively with the following conclusions:

1) The image acquisition method under the shading conditions was proposed, and the image acquisition system with shading function was built up. This method also addressed the issue that many noisy factors such as the natural light, bright spots and reflection in the background impacted the positioning accuracy of rice seedlings.

2) The positioning methods of comparison between different points and dynamic position were put forward. The images of rice seedlings were processed and segmented through such methods as graying green components, PointFilter filtering noise and automatic threshold. When the seedlings were marked in the binary images, the number of connected areas required to extract the complete rice seedlings was calculated with the identification of complete rice seedlings. The center of rice seedlings was calculated according to the fixed number of connected areas. Based on this method, the positioning methods of comparison between different points and dynamic position were favored to make the positioning more accurate.

3) In order to test the identification and positioning accuracy, the soil bin and field test were conducted respectively. Soil bin testing results were as follows: in 50 image samples with water, the center positioning accuracy of rice seedlings was $8 \mathrm{~mm}$ whereas in 50 image samples without water, the center positioning accuracy of rice seedlings was 9.1 $\mathrm{mm}$. In field tests, the protected domain was marked according to the stem-base diameter of rice seedlings. The results showed that in 50 field image samples, the positioning accuracy was $8 \mathrm{~mm}$ with $90 \%$ positioning accuracy. Besides, it took $8.5 \pm 1.5 \mathrm{~ms}$ to process a single image. Test results made clear that the identification and positioning method based on machine vision technology could meet the technical demands of mechanical weeding in terms of positioning accuracy and processing speed.

\section{Acknowledgments}

This research was supported in part by the Natural Science Foundation of China (No. 51575195), Guangdong Natural Science Foundation (No.2015A030313402), Guangdong Science and Technology Support Plan (No.2014B020207003), the Earmarked Fund for Modern Agro-industry Technology Research System (No.CARS-01-33),Pearl River Nova Program of Guangzhou(No.2014J2200041).

\section{References}

[1] M. Xu, Q. Long, L. Bai, T. Zuting and Z. Yanjun, "Present status and prospects of mechanical weeding equipment and technology in paddy field", Transactions of the Chinese Society of Agricultural Engineering(Transactions of the CSAE), vol. 27, no. 6, (2011), pp. 162-168.

[2] N. D. Tillett, T. Hague, A. C. Grundy and A. P. Dedousis, "Mechanical within-row weed control for transplanted crops using computer vision”, Biosystems Engineering, vol. 99, no. 2, (2008), pp. 171-178.

[3] B. Astrand and A. J. Baerveldt, "An agricultural mobile robot with vision-based perception for mechanical weed control Autonomous Robots, vol. 13 (2002), pp. 21-35.

[4] Z. Chunlong, H. Xiaolong, L. Weidong, Z. Yan, L. Nan, Z. Junxiong and L. Wei, "Information acquisition method for mechanical intra-row weeding robot", Transactions of the Chinese Society of Agricultural Engineering(Transactions of the CSAE), vol. 28, no. 9, (2012), pp. 142-146.

[5] H. Lian, L.Xiwen, Z. Shan, Z. Zhigang, C. Xiongfei and L. Chaoxing, "Plant recognition and localization for intra-row mechanical weeding device based on machine vision", Transactions of the Chinese Society of Agricultural Engineering(Transactions of the CSAE), vol. 29, no. 10, (2013), pp. 12-18. 
[6] A. D. Nakarmi and L. Tang, "Automatic inter-plant spacing sensing at early growth stages using a 3D vision sensor", Computers and Electronics in Agriculture, vol. 82, (2012), pp. 23-31.

[7] D. C. Slaughter, D. K. Giles and D. Downey, "Autonomous robotic weed control systems: A review", Computers and Electronics in Agriculture, vol. 61, (2008), pp. 63-78.

[8] T. Jinglei, C. Xiaoqian, M. Ronghui and W. Dong, "Weed detection using image processing under different illumination for site-specific areas spraying", Computers and Electronics in Agriculture, vol. 122, (2016), pp. 195-202.

[9] C. Jiao, J. Guoquan, D. Shangfeng and K. Xin, "Crop rows detection based on parallel characteristic of crop rows using visual navigation", Transaction of the Chinese Society of Agricultural Engineering(Transactions of the CSAE), vol. 25, no. 12, (2009), pp. 107-113.

[10] C. Qian, W. Ku and L. Han, "Detection algorithm for crop target multi-lines of the field image based on machine vision", Transactions of the Chinese Society of Agricultural Engineering(Transactions of the CSAE), vol. 26, no. 1, (2010), pp. 187-191.

[11] L. Kai, Y. Yanli, L. Kai, G. Song, Z. Qing and Z. Liang, "Determination and grading of Anthurium based on machine vision", Transactions of the Chinese Society of Agricultural Engineering(Transactions of the CSAE), vol. 29, no. 24, (2013), pp. 196-203.

[12] S. Meihong and W. Wenguang, "Fabric Defect Detection Algorithm Based on Blob Algorithm", Modern Electronics Technique, vol. 24, (2010), pp. 29-32.

[13] X. Min, T. Wanyou, M.Qianli and H. Jiangiang, "Research of Printing Defect On-line Detection Based on glob Algorithm", PACKAGING ENGINEERING, vol. 32, no. 9, (2011), pp. 20-23

[14] T. Jihui, "A novel building boundary extraction method for highresolution aerial image", ARCHIVE, vol. 1, no. 2, (2014), pp. 19-22.

[15] Z. Zhibin, L. Xiwen, Z. Ying, H. Fuxiang and X. Xiaodong, "Segmentation algorithm based on color feature for green crop seedlings", Transactions of the Chinese Society of Agricultural Engineering (Transactions of the CSAE), vol. 27, no. 7, (2011), pp. 183-189.

[16] H. Lian, L. Chaoxing, L. Xiwen,Y. Weiwei, X. Yi, Z. Hao and Z. Zhigang, "Design and experiment on auto leveling control system of agricultural implements", Transactions of the Chinese Society of Agricultural Engineering(Transactions of the CSAE), vol. 31, no. 8, (2015), pp. 15-19.

[17] C. Shuren, L. Yixin, M. Hanping, S. Baoguo, Z. Yuzhu and C. Bing, "Research on Distinguishing Weed from Chop Using Spectrum Analysis Technology", Spectroscopy and Spectral Analysis, vol. 29, no. 2, (2009), pp. 463-466.

[18] Y. Zhang and D. C. Slaughter, "Hyperspectral species mapping for automatic weed control in tomato under thermal environmental stress", Computers and Electronics in Agriculture, vol. 77, (2011), pp. 95-104.

[19] Y. Zhang and D. C. Slaughter, "Influence of solar irradiance on hyperspectral imaging-based plant recognition for autonomous weed control”, BioSystems Engineering, vol. 110, (2011), pp. 330-339. 